\title{
Estudio de la relevancia de las revistas latinoamericanas utilizando un factor de impacto renormalizado
}

\author{
ANa Ma. Ramírez Romero \\ ESTHER 0. GaRcía MaNdujano \\ J. ANTONio del Río Portilla \\ Centro de Investigación en Energía, UNAM, A.P. 34, 62580 Temixco, Mor. \\ tel. (52) (73) 250052 , (52) (5) 62297 21, fax (52) (73) 250018 \\ E-mail: antonio@ servidor.unam.mx
}

\section{RESUMEN}

La ciencia en Latinoamérica ha buscado tener un papel cada vez más relevante en el ambiente científico mundial. Uno de los esfuerzos se dirige a la edición de revistas científicas que logren un nivel de calidad reconocido mundialmente y que respondan a los intereses de las comunidades latinoamericanas. La justificación a la existencia de dichas revistas se sostiene fundamentalmente en la creación de espacios adecuados paralas investigaciones regionales y en el desarrollo de una tradición científica latinoamericana. Para que este crecimiento continúe se requiere hacer explícita la relevancia de las revistas científicas latinoamericanas. En este trabajo se usa un Factorde impacto renormalizado, para analizar la evolución temporal dela relevancia de las revistas latinoamericanas que han sido incluidas en el Journal Citation Repats Este factor de impacto renormalizado está definido de tal forma que considera las particularidades de las Categorías del JCRa la que pertenece cada revista analizada. Se revisa la información del periodo 1991-1997. Con esteanálisis encontramos que en general la evolución temporal de la relevancia de las revistas latinoamericanas incluidas en el JCR tiene una tendencia positiva, aunque todavía no impactan de manera importante en sus respectivas áreas del conocimiento.

Palabras clave: Relevancia de revistas latinoamericanas, Factor de Impacto, Revistas científicas 


\title{
A RE-NORMALIZED IMPACT FACTOR STUDY OF THE RELEVANCE OF LATIN-AMERICAN SCHOLARLY JOURNALS \\ ANA Ma. RamíreZ-Romero, Esther O. García-Mandujano J. ANTONio DEL Río-Portilla
}

\begin{abstract}
Latin-A merican science has sought to become ever more relevant in the global scientific community. O ne thrust of this impulse is the creation of world-class scientific journals that respond to the interests of the Latin-American community. Such journals are justified in so much as they open forums for the dissemination of research that is regionally relevant and fuel the development of the Latin-A merican scientific tradition. In order for these journals to continue to prosper, it is important to explicitly describe their impact in the global scientific community. In the present study a re-normalized impact factor is used in order to analyze the evolution of the relevance of Latin-American scholarly journals included in the Journal Citation Reports (JCR) for the period 1991-1997. This re-normalized impact factor is defined in such a way that it takes into account the specificity of the JCR categories under which each journal falls. It was found that for the period 1991-1997 the relevance of Latin-American scholarly journals included in the JCR was generally on the rise, but that they failed to exert significant impact in their respective fields.

Key Words: Relevance of Latinoamerican Journals, Impact Factor, Scholarly Journals
\end{abstract}

\section{INTRODUCCIÓN}

H l trabajo de las comunidades científicas latinoamericanas ha buscado tener un $\perp$ papel cada vez más relevante en el ámbito de la ciencia mundial. Una de las acciones de la comunidad científica latinoamericana que promueve sus logros es la edición de revistas científicas. Éstas emanan en su mayoría de las universidades e instituciones de investigación, y tienen ese carácter científico. Aunque no todas estas revistas poseen igual nivel de calidad, así lo comprobamos al revisar el Jaumal CitationReports(JCR)y encontrar que sólo dieciocho del total de revistas científicas latinoamericanas tienen asignado un factor de impacto. Es de todos conocido que la referencia de un factor de impacto es una herramienta muy valiosa en la evaluación del trabajo científico en el ámbito mundial. Además sabemos que no existe en la región un método de evaluación del trabajo científico que permita tener información retrospectiva y completa de las publicaciones científicas latinoamericanas.

Evaluar cuantitativamente la relevancia de las revistas latinoamericanas en su conjunto dista mucho de ser una tarea fácil. Esta evaluación debe realizarse utilizando los estudios métricos de la información, en particular los de 'bibliometría', rama del quehacer humano definida por Pritchard, ${ }^{1}$ citado por S. G orbea-Portal, ${ }^{2}$ como

1 A. Pritchard.- "Statistical Bibliography or Bibliometrics?" - Jaumal of Doamentation (London) 25(4):348-349, D ecember, 1969. (Citado por G orbea-Portal, S.)

2 S. Gorbea-Portal.- "Principios teóricos y metodológicos de los estudios métricos de la información”.- Investigacón Biblictecoógica 8 (17):23-32, 1994. 
"la aplicación de los métodos matemáticos y estadísticos a los libros y otros medios de comunicación." Una de las labores de esta especialidad es la búsqueday determinación de elementos de juicio para evaluar de la calidad de la producción científica. Sobre el tema de la evaluación de las revistas científicas, existe un amplio trabajo de autores como Luukkonen, ${ }^{3}$ Makino ${ }^{4}$ y Ali. ${ }^{5}$ En particular el de G arfield ${ }^{6}$ (desde 1963), del Institute for Scientific Information (ISI), quea través dela publicación del SaienceCitationIndex y del Jaumal Citation Reparts ${ }^{7}$ nos proporciona herramientas útiles para esta labor. La filosofía valuatoria del JCRse sustenta en que la calidad de un artículo publicado depende de la cantidad de citas que recibe; si una revista contiene artículos que son muy citados es considerada como una revista relevante. A su vez, se dice que un artículo puede ser importante si la revista en la que se encuentra publicado recibe un gran número de citas anuales. Esta información se publica en el JCR. Una de sus siete secciones es una lista alfabética de todas las revistas indizadas con su correspondiente Factor de Impacto $(F)$. A pesar de que existe un gran número de artículos en los que se explican las bondades de esta técnica de evaluación, ${ }^{8}$ estamos convencidos que el factor de impacto no debe ser el único elemento cuantitativo para determinar la calidad de una revista. Por esta razón buscamos una forma de renormalizarlo que permita proceder con nuestro análisis de relevancia.

Antes de abordar el estudio de la evolución de las revistas latinoamericanas recordemos que el factor de impacto de una revista está definido como:

$$
F=\frac{C i}{A r}
$$

donde Ci es el número total de citas que reciben los artículos publicados en ella en los últimos dos años y Ares el número total de artículos que se publicaron en esa misma revista durante esos dos años. Como se explicará posteriormente, considerar este número como un indicador definitivo de la relevancia de la revista en el ambiente científico general no es conveniente, ya que no toma en cuenta las particularidades de cada disciplina. Como una respuesta a esta observación, en el JCR se incluye un apartado por categorías temáticas, esta sección del JCR presenta grupos de revistas por categorías ordenados en forma descendente de acuerdo con su respectivo

3 Terttu Luukkonen.- "Why has Latour's theory of citation been ignored by the Bibliometric community? Discussion of sociological interpretations of citation analysis".- Scientomerics 38 (1):27-37, 1997.

4 J. Makino.- "Productivity of Research Groups - Relation Between Citation Analysis and reputation within research communities".- Saientamerics 43 (1):87-93, 1998.

5 N. S. Ali, Harold C. Y oung and Ali Nasser S. - "D etermining the quality of publications and research for tenure of promotion decisions. A preliminary checklist to assist".- LibraryReview45: 39, 1996.

6 E. Garfield.- "Validation of Citation Analysis".- Joumal of theAmericanSocieyforInfomationSaienc48 (10):962, 1997.; G arfield, E. "Significant journals in science”. - Nature264 (5587): 609, 1976.

7 Jaumal CitationRepats A Bibliometric Analysis of Science Journals in the ISI D atabase. 1994. Saience Citation Index.

8 E. Garfield. Op Cit. 
factor de impacto. D e esta manera, en cada categoría la revista que se lista primero es la que tiene un factor de impacto mayory por ende es una revista relevante en la disciplina o temática de la categoría. Es en este apartado donde apreciamos con mayor claridad que el $\mathrm{F}$ depende fuertemente de las categorías, pues hay categorías donde el F máximo es del orden de 1 y otras con F mayor a 20.

En los trabajos de O rlans, ${ }^{9}$ Makino, ${ }^{10}$ Balaban ${ }^{11}$ y Kostoff 12 sobre medición de la calidad tanto de artículos como de revistas, se analizan los sistemas de evaluación y en especial el que utiliza el ISI en el sentido de cuestionar la validez que tiene medir la calidad de un artículo en función del lugar donde se publica y del número de citas que recibe. Autores como Makino y Balaban consideran además que comparar una categoría con otra no relacionada directamente (por ejemplo biologíay física) es inadecuado, y concluyen que el $\mathrm{F}$ no es objetivo. Kostoff, sugiere evaluar el artículo científico contra las citas que recibe pero considerando las áreas temáticas o categorías a las que pertenece. Él piensa que esta metodología se podría aplicar incluso alas revistas. Hay otras propuestas, por ejemplo Balaban, Luukkonen, o Ali quienes señalan diferentes formas cualitativasy adhooque ajustarían la evaluación a unainstitución o grupo de investigación.

O tros autores consideran que simplemente no se debe utilizar el factor de impacto para medir la calidad de la investigación científica, uno de ellos es Seglen ${ }^{13}$ quien argumenta que los hábitos y dinámica de citación pueden ser diversos en los diferentes campos de investigación, lo que hace que la comparación valuatoria sea difícil o imposible. Para enfatizar esteaspecto se refiere al trabajo deMoed:14 "Elimpacto de citación de un campo de investigación es directamente proporcional al número medio de referencias por artículo, que varía considerablemente de área en área (es dos veces más alto en bioquímica que en matemáticas, por ejemplo)". En tanto que Hamilton argumenta: 15 "en las humanidades y en las artes las citas a artículos se utilizan muy raramente, haciendo que estos campos de investigación (y otros) queden virtualmente sin citas." O curre algo parecido incluso en áreas de investigación más dinámicas como bioquímica y biología molecular, en donde se publican reportes que rápidamente se vuelven obsoletos pero que logran un gran número de citas, en contraste con áreas en las que la vida de la literatura es más larga, como en matemáticas

9 Harold O rlans. - "The world's scientific papers".- Change29 (3):6, 1997.

10 J. Makino. Op Cit.

11 A. T. Balaban.- "How should citations to articles in high - and low - impact journals be evaluated, of what is a citation worth?".- Sientomerics37 (3):495-498, 1996.

12 R. N. Kostoff. - "Citation analysis cross-field normalization: a new paradigm".- Säentamerics39 (3):225-230, 1997.

13 P. O. Seglen.- "Why the impact factor of journals should not be used for evaluating research". BMJ: BritishMeeical Jaumal 314 (7079):498, 1979.

14 H. F. Moed.- "The application of bibliometric indicators: important field-and time-dependent factors to be considered".- Säentomtrics 8:177-203, 1985. (Citado por P.0. Seglen).

15 D.P. Hamilton.- "Research papers: who's uncited now".- Säene251: 25, 1991 (Citado por P.O. Seglen). 
que tiene un índice de citas menor, lo que provoca que el factor de impacto sea bajo para esta materia. También argumenta quela evaluación que haceel ISI es deficiente, ya que no separa entre las revistas que publican muchos artículos de revisión 0 'notes', documentos que generalmente interesan mucho a los investigadores y son muy citados, y las revistas que generalmente publican más artículos y menos 'notes'; considera que éstas últimas están en desventaja.

Coincidimos en que el sistema propuesto por $\mathrm{G}$ arfield no es el más adecuado. Sin embargo, se utiliza en los procesos de evaluación debido a que, hasta ahora, es el sistema que más influencia tiene en la valoración del trabajo de la comunidad científica internacional y difícilmente va a ser sustituido por otro que no logre cubrir las áreas temáticas, las revistas, la información retrospectiva, etcétera, que es lo que justifica suficientemente su confiabilidad.

Buscando sobrepasar en alguna medida estas limitaciones, recientemente se propuso un factor de impacto renormalizado ${ }^{16}$ que da por resultado un número que considera la particularidad del campo de conocimiento y, por ende, de la categoría a la que pertenece cada revista de acuerdo con el JCR. Es un nuevo parámetro, Fr, basado en las definiciones de parámetros adimensionales en ingeniería. ${ }^{17}$ En este trabajo se analiza la evolución temporal de la relevancia de dieciocho revistas latinoamericanas incluidas en el JCR utilizando este Fr.

En esta parte exponemos lo que sucede en el caso de las revistas latinoamericanas, ya que todas ellas se encuentran en diferentes categorías; y además, para hacer evidente la imposibilidad de realizar una comparación directa, a continuación analizamos el factor de impacto.

Las revistas latinoamericanas incluidas en el JCR, están ubicadas en las siguientes categorías: astronomía y astrofísica, biología, ciencias multidisciplinarias, física, genética, medicina, microbiología, agricultura, investigación médica y experimental y zoología, como se observa en la Tabla 1. Una comparación directa del factor de impacto de estas revistas no puede darnos un parámetro cuantitativo libre de interpretaciones subjetivas. Para ilustrar este problema analicemos los factores de impacto de estas revistas en los últimos siete años en la Tabla 2.

Notamos que no es posible la comparación directa entre estas revistas, tomando en cuenta que las temáticas son completamente diferentes y que su materia de trabajo no es equiparable; aún así tratamos de inferir un análisis con sólo estos datos. Al observarlos apreciamos que las revistas RMAA, RCHN y BJMBR son las revistas latinoamericanas que tienen un mayor factor de impacto, por lo que estaríamos tentados a decir que son las más relevantes en el ambiente científico de la región. Podríamos mencionar que la revista RMAA tiene importantes fluctuaciones

16 A.M. Ramírez, E.O . GarcíayJ. A. del Río. "Renormalized Impact Factor" aceptado en Säentanetrics

17 - S. Cuevas, E. Ramos. - "Heat transfer in a MHD chanel flow with boundary conditions of the third kind".- Applied SaienceReserch48:11-33, 1991.

- H. Schlichting. - Baundary-Layer Theary, Mc Graw-Hill, New Y ork, 1979.

- A.M. Ramírez, E.O. G arcía y J. A. del Río. OpCit. 
a lo largo de estos años, y que con la información que tenemos y en este trabajo no podemos determinar a que obedecen; lo que sí podemos señalar es que en una evaluación seria no se aceptaría calificarla como de buena calidad en los años 1993, 1996 y 1997, y de menor impacto en 1992, etcétera. Con estos datos no podemos determinar si la revista ha logrado consolidarse o si más bien sólo se mantiene en circulación, etcétera. Por otro lado, la revista RMC es de las que presenta un F constante, sin variaciones bruscas en el tiempo, pero la información que podemos obtener es igualmente incompleta o parcial y estos datos no determinan si este comportamiento en su F es favorable para la revista al considerarla en el contexto científico de su categoría. En cambio aunque las revistas de zoología y biología han tenido ascensos sustanciales en su Fa lo largo de estos últimos años parecen crecer también en importancia. Pero si queremos hablar de la RBG, RBT o INTER es aún más complicado porque presentan más variación en el $\mathrm{F}$ en todos los años y no podríamos afirmar qué tan relevantes son en el ámbito científico ni emitir un juicio sobre su evolución temporal, ya que los datos muestran severas fluctuaciones año con año. En la revista RMF, que se incluyó en el JCRa partir de 1994, se observa que su F varía alrededor de 0.2; se nota que se encuentra entre las cuatro revistas con F más alto en 1996, pero realmente no podemos emitir un juicio sobre su relevancia científica. D e manera general podemos mencionar también que la CJAC apareció en el JCR sólo los años 1992, 1996 y 1997, con F muy bajos, pero tampoco podemos afirmar que es poco relevante. Podríamos mencionar además los años en que cada revista ha tenido un F mayor, pero esto no enriquecería nuestra evaluación. Para analizar de una forma global y dinámica la evolución temporal de estos datos es conveniente presentarlos en forma gráfica. En la gráfica 1 se muestran los factores de impacto de las revistas mencionadas. Ahí podemos notar que el F presenta para todas las revistas cambios de un año a otro, y en algunos casos fluctuaciones muy pronunciadas. La mayoría de las revistas latinoamericanas tienen un factor de impacto menor a 0.4; pero este número no puede ser indicativo debido a que todas ellas pertenecen a diferente categoría. En particular, RMAA en los últimos años tiene el mayor factor de impacto, pero sería apresurado tomarla como la revista más relevante de la región. Sin embargo, las demás revistas parecen presentar un comportamiento muy compacto, aunque errático y no es posible compararlas directamente. Basándonos en esta gráfica no podemos emitir un juicio comparativo entre las dieciocho revistas debido a que, como ya mencionamos, se presentan diferentes comportamientos de los factores de impacto en cada una de las categorías y se acepta que esta situación afecta directamente al $\mathrm{F}$. La revista que más recientemente tiene un factor de impacto es CM.

También incluimos dos tablas - una con los factores de impacto máximos (Tabla 3) y otra con los datos de la mediana (Tabla 4) para cada una de las categonías a las que pertenecen las revistas analizadas- , con datos complementarios que nos permitirán observar claramente el comportamiento de cada categoría. 
Tabla 1.

Reistas científicas latinoamericanasy su categráa temática.

\begin{tabular}{|c|c|c|}
\hline Revista & Clave & Categonías \\
\hline Rev. Mex. deAstranamíayAstrouísica & RMAA & Astron. and astrophys \\
\hline Rev. Mex. deFísica & RMF & Physics \\
\hline Rev. Médica deChile & $\mathrm{RMC}$ & Medicine general and internal \\
\hline Rev. DeBidoǵa Tropical (Costa Rica) & RBT & Biology \\
\hline Rev. Brasileira deGenética & RBG & Genetics and heredity \\
\hline Rev. Chilena deHistaria Natural & $\mathrm{RCHN}$ & Multidisciplinary sciences \\
\hline Rer. DeMiđadaidoǵa (Brasil) & RM & Microbiology \\
\hline CubanJaumal of Agiaultural Saience & CJAS & $\begin{array}{l}\text { Agriculture } \\
\text { Agriculture dairy and animal }\end{array}$ \\
\hline Interciencia & INTER & Multidisciplinary sciences \\
\hline $\begin{array}{l}\text { Jaumal of agiaulture(Univ. Puato } \\
\text { Riø) }\end{array}$ & JA & Agriculture \\
\hline Mediana (BuenosAires) & MED & Medicine general and internal \\
\hline Memnias dbInstitutoOsualdbCruz & MIOC & $\begin{array}{l}\text { Medicine, research and experi- } \\
\text { mental }\end{array}$ \\
\hline Nematrópica (Maracay, Ven) & NEM & Zoology \\
\hline Pesquisa Agropeuaria Brasileira & PAB & Agriculture \\
\hline Revista deInvestigación Clínica & RIC & Medicine general and internal \\
\hline $\begin{array}{l}\text { BrazilianJ. of Meclical andBiological } \\
\text { Research }\end{array}$ & BJMBR & $\begin{array}{l}\text { Medicine, research and experi- } \\
\text { mental }\end{array}$ \\
\hline Brazilian Jaumal of Gentics & BJG & Genetics and heredity \\
\hline CieniasManinas & $\mathrm{CM}$ & Marine and freshwater biology \\
\hline
\end{tabular}




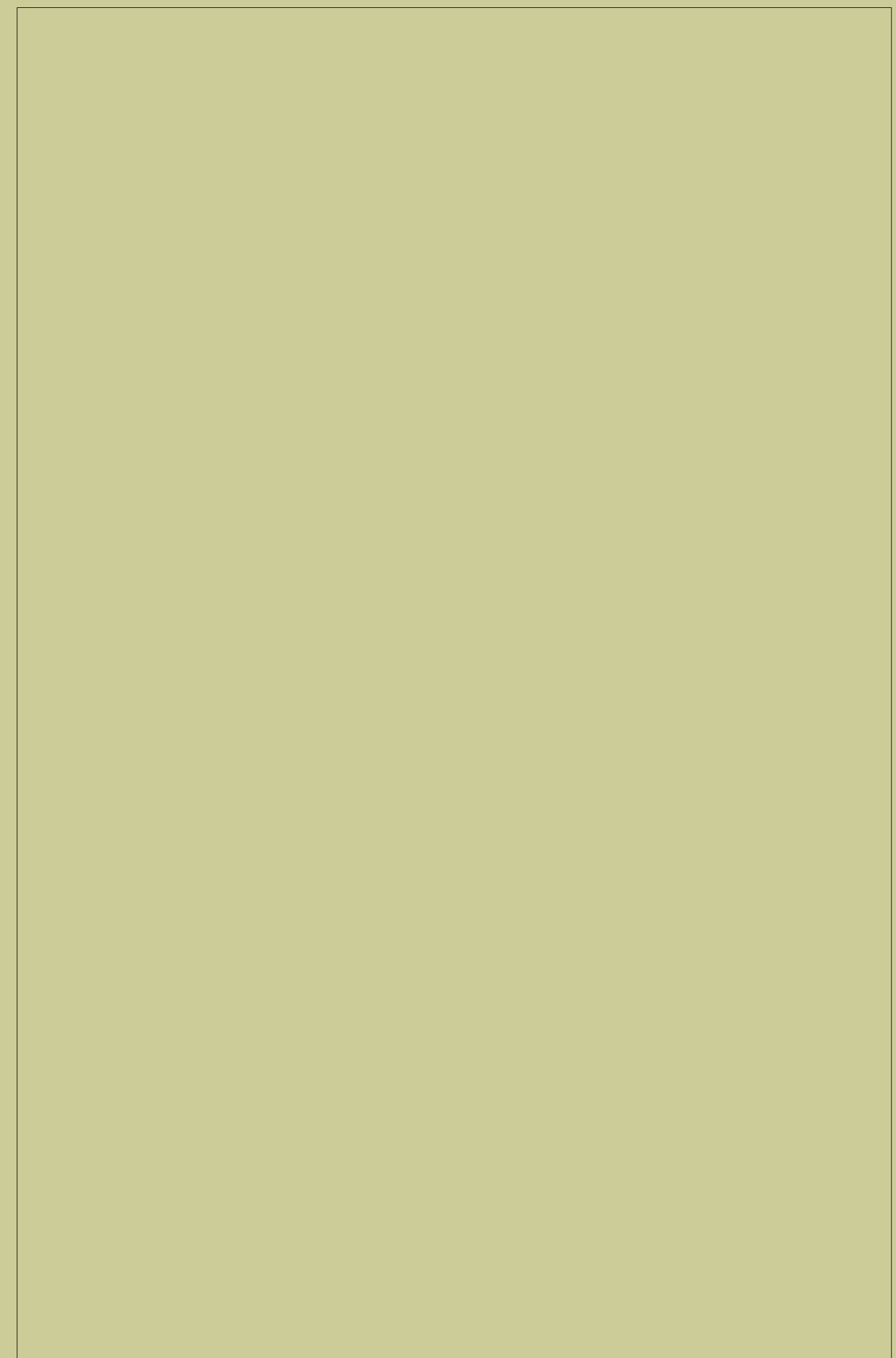


Al comparar los datos de la Tabla 2 con los de la Tabla 3 vemos enfatizada la dependencia de los factores de impacto en el ámbito de las categorías. Los datos coresponden a los factores de impacto máximos de cada una de las categorías que incluyen alas revistas seleccionadas. Como se puede apreciar, los factores máximos en cada una de las categorías también oscilan y presentan un comportamiento que debe ser considerado en la evaluación de las propias revistas latinoamericanas.

0 tro indicador de las categorías es el factor de impacto que ocupa la mediana en la sección del JCR por categorías. Mostramos estos datos en la Tabla 4.

O bservemos que también los valores de la mediana oscilan; sin embargo muestran un comportamiento uniforme entre las diferentes categorías. Además las medianas son números cercanos a los valores de la Tabla $2(\mathrm{~F})$.

Nuevamente tenemos que al evaluar sólo con estos datos, lainformación que obtenemos continúa siendo cualitativa. En la Tabla 3 vemos claramente que la comparación directa de los factores de impacto es desventajosa para aquellos que trabajan en las áreas de agricultura, zoología y astronomía, ya que los factores de impacto máximo en estas áreas son los más bajos; en tanto que con los datos de la Tabla 4, para la categoría de medicina general, un factor de impacto cercano a 0.5 puede representar estar ubicado entre los valores medios de las revistas en cuanto a relevancia. Con esto, nos queda claro que el factor de impacto es fuertemente dependiente del área de conocimiento del estudio realizado.

Al tomar esta información separadamente podemos darnos cuenta de que elJCR es una herramienta muy valiosa parala evaluación del trabajo científico, pero no permite hacer un juicio directo dela calidad delas diferentes revistas incluidas en él ni de cada una en particular.

\section{MODELO}

En esta sección, con el afán de hacer este artículo autocontenido, presentamos la metodología para calcular el factor de impacto renormalizado ${ }^{18}$ y discutimos sus bondades. Como ya se mostró en cada categoría se tiene una revista con un F máximo (Fmax, Tabla3) y fácilmente podemos obtener la mediana de las revistas de la misma categoría (Fmæd Tabla 4), de tal manera que se puede construir un nuevo número para cada revista perteneciente a la categoría de la forma. ${ }^{19}$

$$
F c=\frac{(F-F m e d)}{F \text { max }-F m e d}
$$

18 A.M. Ramírez, E.O. García y J. A. del Río. Op.Cit

19 Ibid 
donde Fces el nuevo número de la revista considerando su categoría y F es el factor de impacto de la revista. Este parámetro renormalizado frecuentemente se llama en ingeniería 'parámetro reducido. ${ }^{20} \mathrm{E}$ ste nuevo número es realmente un factor de impacto renormalizado con la diferencia entre el factor de impacto máximo y el factor de impacto que ocupa la mediana de cada categoría. Claramente este nuevo número es 1 para la revista con máximo $\mathrm{F}$ dentro de la categoría y es 0 para la revista con el $\mathrm{F}$ que ocupa la mediana. Mientras que un Frimplica que la revista ocupa un lugar de menor importancia relativa al máximo de la categoría. Con este número se pueden comparar directamente revistas de una categoría con revistas de otra. ${ }^{21}$

El factor de impacto renormalizado(Fd servirá para definir unívocamente a las revistas que se encuentran dentro del rango entre la revista que ocupa el lugar de la mediana en la lista de la categoría y el máximo, F>0. Con esto se pueden presentar los resultados claramente, incluso se podrá sugerirle a los investigadores que envíen sus trabajos para publicación a las revistas con F $>0$. Aquí surge una pregunta ¿qué sucede cuando una revista aparece en dos o más categorías? Con el afán de tener un solo número se podría obtener el promedio de los factores renormalizados. ${ }^{22}$

$$
F r=\frac{\sum F c i}{n}
$$

donde Faies el factor renormalizado de acuerdo con la categoríaiy nindica el número de categorías donde se encuentra listada la revista. D e esta forma el valor Frasociado a una revista puede ser comparado directamente con el valor de otra revista, aun y cuando las revistas sean de diferente campo de conocimiento. Claramente si la revista está en una sola categoría $\mathrm{Fr}=\mathrm{Fc}$ Con este nuevo indicador a cada revista se le asocia un número renormalizado que permite el análisis comparativo entre diferentes áreas y que considera simultáneamente la particularidad de los campos de conocimiento. Con este número podemos comparar la relevancia de las revistas latinoamericanas aunque todas ellas se dediquen a publicar trabajos en diferentes campos del conocimiento.

\section{ANÁ LISIS COMPARATIVO DE LAS REVIST AS LATINOAMERICANAS USAN - DO UN FACTOR DE IMPACTO RENORMALIZADO}

En esta sección mostramos un ejemplo comparativo donde el factor de impacto renormalizado introduce de manera natural elementos nuevos y claros en la apreciación de la relevancia de las revistas latinoamericanas. En la Tabla 5 se presentan los factores de impacto renormalizados para dichas revistas; con estos datos cambia radicalmente nuestra primera evaluación.

\footnotetext{
20 - S. Cuevas y E. Ramos. Op Cit.

- H. Schlichting. Op Cit.

21 A.M. Ramírez, E.O. García y J. A. del Río. Op Cit

22 Ibid
} 
Lo primero que observamos es que sólo cuatro valores, dos de la revista INTER, en 1992 y 1993, uno de la revista NEM, en 1991 y uno de la revista RMAA en 1997, son positivos. Esto es resultado primero de que, como ya se explicó, en esos años tienen un Fentre el Fmax y el Fmeddentro de sus categorías. Por otro lado esto podría indicar que estas tres revistas publicaron en esos años algún o algunos artículos que contribuyeron al desarrollo de algún tema de actualidad o avance sustancial en esas áreas de investigación, pero en los siguientes años esos trabajos ya no fueron citadosy la revista vuelve a obtener un F por debajo del valor de mediana en la categoría que la incluye. Pensamos que estos repuntes positivos en los F's de algunas revistas, podrían ser material de investigación para los editores, ellos podrían obtener información valiosa que les indicara si deben replantear sus políticas editoriales.

Las demás revistas obtuvieron un Frpor debajo de la mediana de sus categorías y esto es el principal indicador de que las revistas latinoamericanas no tienen todavía un papel relevante en la divulgación del trabajo científico en el ámbito mundial. Para hacer un análisis global deesta información analicemos los resultados en función del tiempo en una perspectiva gráfica.

En la gráfica 2 se tiene claramente una panorámica global del comportamiento de las revistas. Aquí notamos cómo la línea correspondientea RMAA que se colocaba por encima de todas las demás, aparece ahora con sus grandes fluctuaciones en el tiempo, pero en una posición que permite compararla con el resto, y podemos concluir que efectivamente es sólo en los últimos dos años de este análisis cuando figura como la revista latinoamericana de mayor Fren el ámbito científico internacional. Sería interesante revisar por qué hay tanta variación en sus valores, comportamiento que no se ve en ninguna otra revista latinoamericana. 0 tro aspecto a notar es que al contar con los datos renormalizados ubicamos inmediatamente cuál es la revista que juega el papel más relevante en su área temática. Las revistas se separan claramente para su mejor comparación y se puede emitir un juicio en cuanto a la relevancia en el tiempo de cada una en la o las categorías respectivas. En el caso de las revistas analizadas aquí, la gráfica 2 muestra claramente cuál puede considerarse la revista latinoamericana de mejor calidad en el ambiente científico de la región, pero vista desde el contexto científico global. En otras palabras, en la gráfica 1 no podemos comparar directamentelas revistas, ya que se aglutinan entre valores muy próximos, o se separan drásticamente del grupo, lo que provoca que se emitan juicios que en realidad no denotan el nivel de calidad de la publicación. En cambio en la gráfica 2 podemos compararlas y observar las características particulares de cada una. Por ejemplo vemos claramente que RCHN, MED, INTER, RIC, BJMBRy RMC tienen un comportamiento más establey con valores en mejor posición relativa ala categoría o área específica a la que pertenecen. Un caso que merece mención es el de la RBG, pues aunque su Frestá entre los más bajos de este estudio se trata de una revista que desde su inclusión en el JCR ha tenido una relevancia ascendente. También gracias a esta gráfica resulta claro cuáles revistas presentan variaciones que deben ser motivo 
de revisiones especiales del tema. En cuanto a la revista CMse observa su reciente inclusión y que dentro de su categoría dista mucho de ser relevante.

Por último debemos mencionar un aspecto que debe animar a la comunidad científica latinoamericana a seguir editando revistas científicas, y es que del total de revistas analizadas sólo 4 presentan una tendencia negativa, lo que nos llevaría a pensar que la tendencia en general dela edición de revistas científicas en Latinoamérica va adquiriendo mayor relevancia.

\section{figura 1}

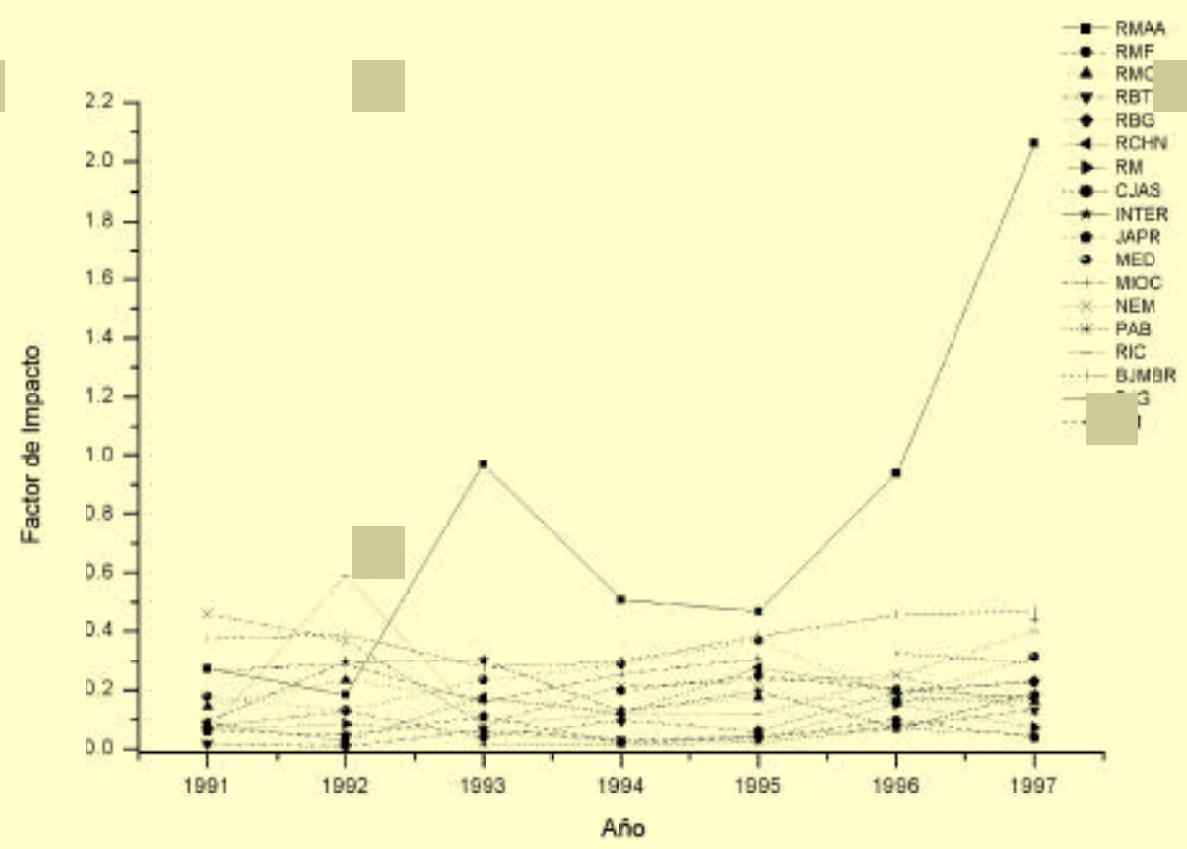


En la gráfica 2 observamos que la mitad de las revistas se agrupa cerca de la mediana con una ligera tendencia negativa. El resto está por debajo de la misma y presenta un comportamiento con fluctuaciones importantes. En la gráfica 3 eliminamos las seis revistas que se alejaban más de la mediana y notamos que el $66 \%$ de las revistas latinoamericanas analizadas presentan en los últimos años un descenso en el Fr. Cuatro revistas se mantienen con tendencia positiva (RMAA, RBT, RBGy RIC).

D e aquí se infiere otra de las bondades del Fr, la detección de fluctuaciones colectivas de las categorías. En particular si los factores de impacto renormalizados delas revistas de una determinada categoría involucraron simultáneamente una moda 0 una temática explosiva, el Frabsorbe y detecta esta situación.

\section{CONCLUSIONES}

$\mathrm{Al}$ analizar las revistas latinoamericanas incluidas en el JCR, utilizando un factor de impacto renormalizado ( $F n$ ), se facilita la determinación de la relevancia en el campo específico de cada revistay la tendencia de estas relevancias. La renormalización se realiza para considerar de una forma cuantitativa las particularidades de cada catego ría en la que el JCR ha dividido las áreas temáticas en las que trabaja la comunidad científica mundial. Considerando estas particularidades y emitiendo un número se compararon directamente revistas listadas en diferentes categorías, sin la necesidad de invocar argumentos externos al factor de impacto renormalizado que consideren el diferente comportamiento de las áreas de investigación.

Uno de los resultados observados con el Fres la estabilidad de la relevancia de las revistas latinoamericanas en los años de este estudio. Hecho que por sí mismo justifica e invita a promover la publicación de estas revistas. También como resultado de este análisis debemos exhortar a los científicos latinoamericanos a leer, utilizary por ende citar más los trabajos de los colegas de la región, hecho que favorecerá el aumento del Fy del Frde estas revistas. Por otro lado es importante que las revistas que se publican y no están incluidas en el JCR, mejoren sus características generales para integrarse al grupo de revistas que difunden los logros del trabajo científico regional entre los grupos de investigadores del mundo.

El JCR no es la única forma de evaluar las revistas, pero sí es la herramienta mayormente utilizada para esta tarea en el ambiente científico internacional y por ende tener un factor de impacto renormalizado para medir la calidad del trabajo científico en general es un elemento de gran relevancia en Cienciometría porque consideramos que enriquece el JCR.

Este factor de impacto renormalizado también resultará útil para o tra clase de estudios de evaluación del conocimiento científico; por ejemplo un estudio de las variaciones bruscas de algunas revistas a través del tiempo, variaciones que pueden ser originadas en modas o apariciones de temas nuevos. 


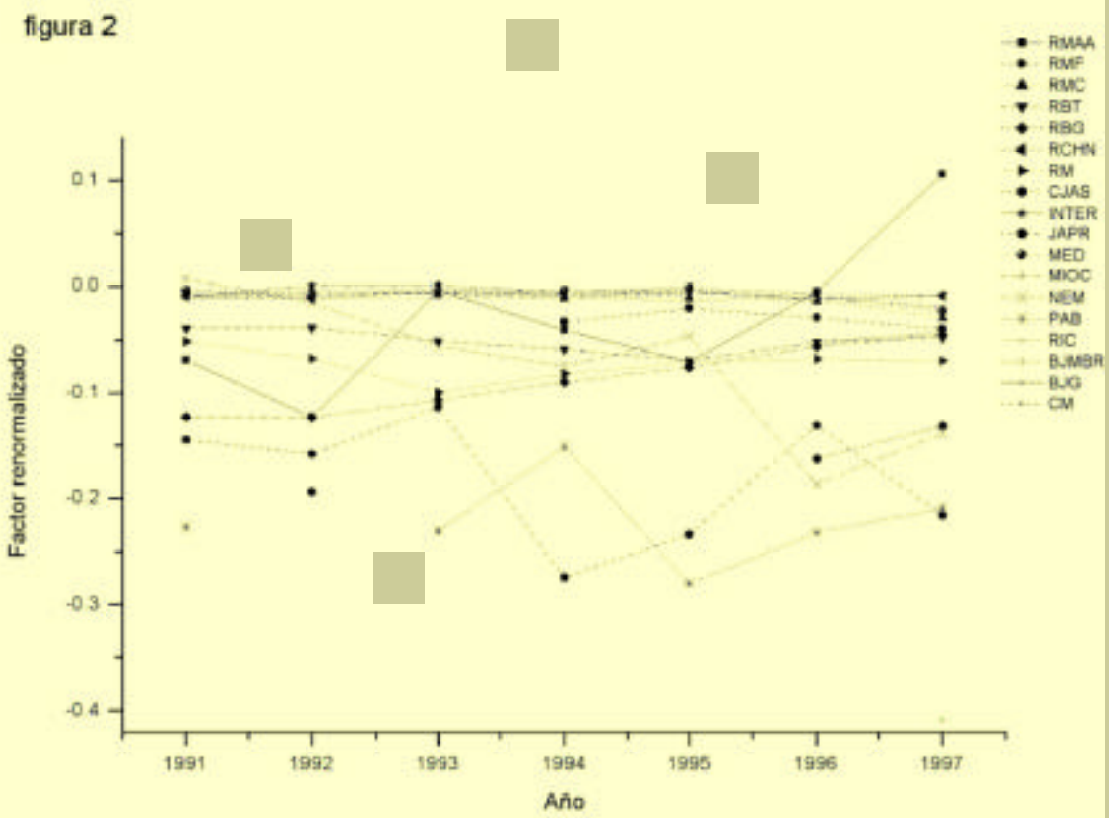

figura 3

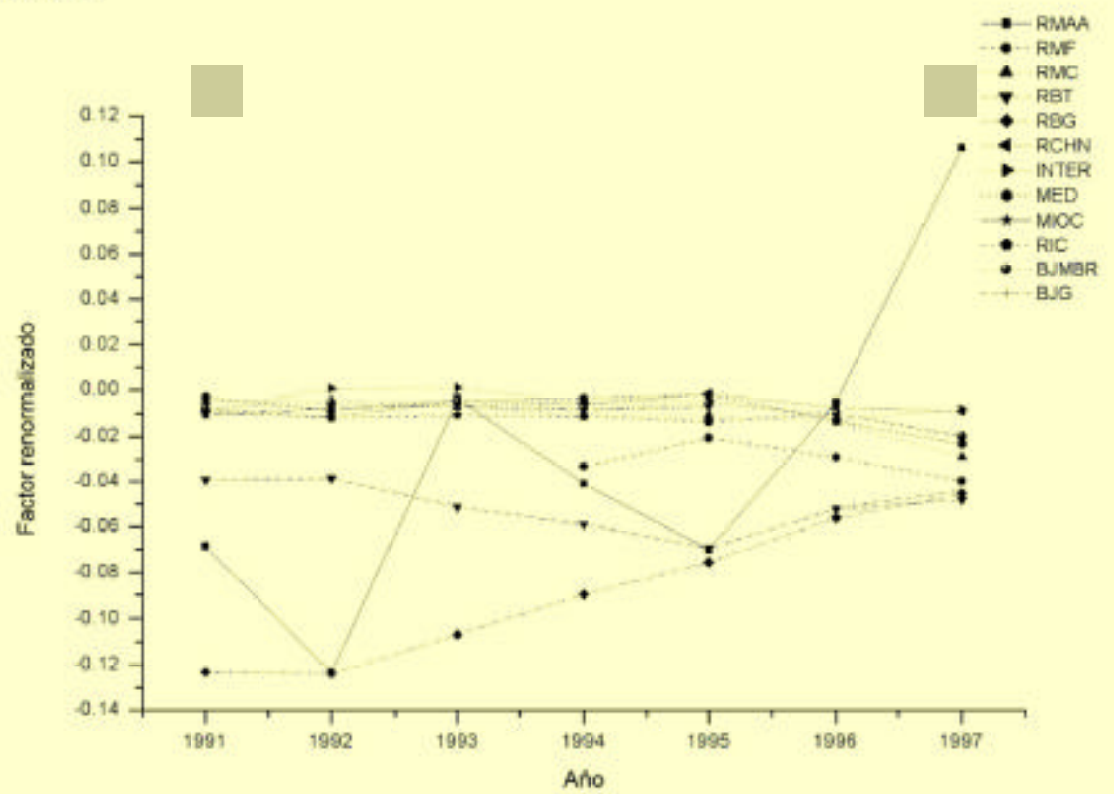

W al índice

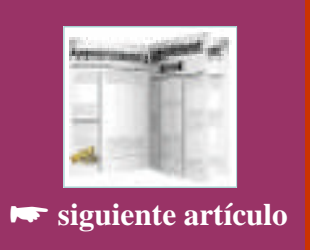

